\title{
Incidencia de la innovación en la exportación de joyas de plata en Perú
}

\section{Incidence of innovation in the export of silver jewelry in Peru}

\section{RESUMEN}

El objetivo de este artículo fue evaluar en qué medida la innovación incide en las exportaciones de joyas de plata de Perú desde el 2015 hasta el año 2019. Para ello, se realizó un estudio no experimental correlacional mediante entrevistas a empresas que, en su conjunto, representaron el $67 \%$ de exportaciones de joyas en plata de Perú al mundo. Los resultados permitieron concluir que la innovación incidió de manera positiva en las exportaciones de joyas de plata de Perú, al aplicar métodos de información tecnológica en relación al uso y procesamiento de datos en el año 2016; así mismo, al considerar desarrollo de productos y procesos de negocio a futuro en los años 2015 y 2019, a condición de que dichas actividades tengan como base la investigación. En consecuencia, el aumento de exportaciones del sector joyería de plata, se asocia a la capacidad de gestión de las empresas para introducir estrategias que permitan desarrollar innovación en procesos específicos para alcanzar sus metas en un mercado global.

Palabras clave: Innovación; exportación; joyas de plata.

\section{ABSTRACT}

The objective of this article was to evaluate the extent to which innovation affects Peruvian silver jewelry exports from 2015 to 2019. For this, a non-experimental correlational study was carried out through interviews with companies that, as a whole, represented $67 \%$ of silver jewelry exports from Peru to the world. The results allowed to conclude that the innovation had a positive impact on the exports of silver jewelry from Peru, by applying technological information methods in relation to the use and processing of data in 2016; Likewise, when considering the development of products and business processes in the future in the years 2015 and 2019, provided that said activities are based on research. Consequently, the increase in exports of the silver jewelry sector is associated with the

\section{Maribel Carruitero} Honores $^{1}$

maribel.carruitero@unmsm.edu.pe

Juan Victoriano Castillo $\operatorname{Maza}^{1}$

jcastillom@unmsm.edu.pe

${ }^{1}$ Universidad Nacional Mayor de San Marcos, Facultad de Ciencias Administrativas. Lima, Perú

Presentado: 25/03/2021 - Aceptado: 31/03/2021 - Publicado: 17/06/2021

(c) Los autores. Este artículo es publicado por la revista Gestión en el Tercer Milenio de la Facultad de Ciencias Administrativas Universidad Nacional Mayor de San Marcos. Este es un artículo de acceso abierto, distribuido bajo los términos de la licencia Creative Commons Atribución 4.0 Internacional (CC BY 4.0) [https://creativecommons.org/licenses/by/4.0/deed.es] que permite el uso, distribución y reproducción en cualquier medio, siempre que la obra original sea debidamente citada de su fuente original. 
management capacity of companies to introduce strategies that allow the development of innovation in specific processes to achieve their goals in a global market.

Keywords: Innovation; export; silver jewelry.

\section{INTRODUCCIÓN}

Según reporta el Banco Mundial (2020), hace más de medio siglo las materias primas han pasado de representar el 30\% del Producto Interior Bruto (PIB) a tan solo 4\%. Este escenario demuestra la necesidad de incrementar la eficiencia en la obtención de productos con valor agregado, siendo necesario para ello la aplicación de la innovación. Así mismo, las empresas que poseen una sólida fuente de ventajas competitivas tienen más probabilidades de sobrevivir y lograr un rendimiento superior. Sobre esta base, cabe esperar que la pequeña y mediana empresa (PYME) innovadora crezca más rápido y sean más eficientes que las no innovadoras en los mercados de exportación (Ratten, 2015).

La innovación es proyectada como la modificación de aspectos ya existentes, mediante mejoras o creando nuevos que impacten favorablemente en el mercado, es así que la Cámara de Comercio de España (CCE, 2020), hace referencia que la innovación es una clara respuesta a los desafíos económicos actuales, particularmente a la hora de competir en un mundo globalizado, sin fronteras.

Por otro lado, las exportaciones, entendidas como el conjunto de bienes y servicios vendidos por un país en territorio extranjero para su utilización, muestran características peculiares en los países de América Latina; en tal sentido, según la Organización para la Cooperación y el Desarrollo Económico (OCDE, 2019), menciona que la perspectiva económica para América Latina registra niveles bajos de productividad en todos los sectores, debido a su enfoque en materias primas sin valor agregado para la exportación. En este contexto, Perú no es la excepción, ya que el ser un país con altas reservas de plata en el mundo, como se evidencia en el Servicio Geológico de los Estados Unidos (USGS, 2020), lo ha hecho vivir un sueño de país exportador, el cual podría terminarse en unas décadas, según lo indica (Rachel
N, 2014), siendo necesaria la creación de economías alternativas como ciertos productos con valor agregado en minería, requiriéndose un mayor enfoque en la innovación para tener mayor probabilidad de éxito. Hay que mencionar además que, según Frohmann et al. (2016), se produce una secuencia del tipo innovación-exportación-innovación, la misma que se pone en marcha cuando las empresas desarrollan una actividad innovadora aprovechando la experiencia obtenida al operar en mercados externos exigentes.

En relación al sector de joyería en el mundo, según Serrano et al. (2020) se evidencia un modo de accionar en la industria de la joyería de plata en países líderes mundiales, como es el caso de la India y Tailandia, quienes adiestran mano de obra especializada y se constituyen en zonas clusters, donde interactúan compartiendo conocimientos acumulados que les permite innovar y les hace más competitivos a nivel internacional.

En nuestra región, países referentes en la exportación de joyas de plata están considerando el accionar de los países líderes. Así, por ejemplo, según la Cámara de Comercio de Bogotá (CCB, 2020) Colombia ha creado clúster en joyería basada en la innovación y el conocimiento, integrando a más de mil quinientas empresas productoras de joyería, generando más de cinco mil empleos, desarrollando herramientas de gestión del ADN y conocimiento del consumidor. Así mismo, México, quién además de adecuar lineamientos y cadenas de valor de sus socios comerciales, crean herramientas y políticas favorables donde la innovación en tecnología de información a jugado un rol elemental en el éxito de sus exportaciones. Cámara de Comercio de Lima (CCL,2020).

Con respecto a Perú, según el Ministerio de Comercio Exterior y Turismo (MINCETUR, 2013), entre los años 2003 al 2013 de cada 10 empresas nacionales exportadoras de productos 
con valor agregado que inician operaciones en un periodo dado, cinco se retiran el siguiente año y solo una pasa los 10 años. Esta problemática se extiende al sector exportador de joyas de plata. De hecho, entre los años 2015 al 2019 según Trade Map (2020), Perú evidencia una escasa representación, con el $0.06 \%$ del total de exportaciones mundiales.

Según la Superintendencia Nacional Tributaria (SUNAT) en el periodo de estudio, tres de las principales empresas exportadoras de joyas de plata están de baja de oficio y una está en proceso de liquidación. Asimismo, tenemos convenios comerciales con 56 países, pese a ello, nuestro mercado real se concentra en solo dos países: Estados Unidos (57\%) y Chile (30\%) (SUNAT, 2020). Al respecto, Oppenheimer (2006) menciona que "el motor que hace avanzar a los países que progresan en la economía global del siglo XXI no es simplemente firmar acuerdos de libre comercio, sino ser más competitivos" (p. 25). En ese sentido, la innovación no sería una opción sino una necesidad para ser competitivos en mercados globales.

En cuanto al accionar de las empresas los últimos años. Los autores (Collier y James, 2016) muestran cambios evolutivos que han tenido que adoptar para estar alineadas en el comercio de mercados globales; sin embargo, esta es una evolución por la que no han pasado las organizaciones de nuestro medio, que han pretendido saltar directamente a herramientas más actuales, sin tener aún los fundamentos ni la cultura de procesos facilitadores de innovación. Es por ello, que en esta investigación se considera la propuesta del manual de Oslo sobre innovación en productos, dado que implican la aplicación práctica de información y conocimiento que puedan influir en cómo una empresa crea utilidad para los clientes; y la innovación de procesos comerciales, que implican el cómo fabrica, comercializa o se establece el precio (OCDE, 2018).

Por todo lo señalado, el presente artículo consideró los siguientes objetivos:

- Determinar de qué manera incide la innovación en la exportación de joyas de plata en el Perú durante el periodo 2015 al 2019.
- Determinar la incidencia de innovación en producto en la exportación de joyas de plata en el Perú.

- Determinar la incidencia de innovación en procesos comerciales en la exportación de joyas de plata en el Perú.

\section{MÉTODOS}

Este artículo tiene un enfoque cuantitativo, de tipo y diseño correlacional, no experimental. La población estuvo constituida por 80 empresas exportadoras de joyas de plata del Perú que según la SUNAT representaron el $100 \%$ de las exportaciones de Joyas de Plata en el periodo de estudio (2015-2019). Se seleccionó una muestra mediante muestreo no probabilístico por juicio (Malhotra, 2008, p. 343), que estuvo constituida por siete empresas, las cuales representaron el 67\% de las exportaciones de Joyas de Plata en el periodo de estudio.

La exportación de joyas de plata fue medida considerando la base de datos de la SUNAT, donde se obtuvo la información de cada empresa seleccionada. Para medir la innovación en productos considerando los ítems: bienes y servicios; de igual modo, la innovación en procesos comerciales considerando los ítems: producción de bienes y servicios; distribución y logística; marketing, ventas y servicios post venta; tecnologías de información; administración y gestión; desarrollo de productos y procesos de negocio, se elaboró un cuestionario estructurado en la escala de Likert, que fue validado por juicio de expertos, que a su vez mostró alta confiabilidad (Alfa de Cronbach = 0.89).

\section{RESULTADOS}

Mediante el presente artículo se evaluó la incidencia de la innovación en la exportación de joyas de plata en siete de las principales empresas de Perú que exportaron en los años 2015, 2016, 2017, 2018 y 2019.

$\mathrm{Al}$ evaluar las puntuaciones globales de la incidencia de innovación en producto en la exportación de joyas de plata en el Perú, se encontró ausencia de relación significativa entre ambas variables en estudio ( $p>0.05)$, tanto para los componentes bien como servicio. 
En cuanto a la incidencia de la innovación en procesos comerciales en la exportación de joyas de plata en el Perú, en la mayoría de los casos, no se encontró una relación estadísticamente significativa entre las variables en estudio ( $p>0.05$ ); sin embargo, al enfocarnos en los ítems a detalle, se encontró lo siguiente: (1) relación directa entre innovación en tecnología de información, en el ítem base de datos y procesamiento de datos, con las exportaciones reportadas en el año 2016 ( $\mathrm{r}=0.767, \mathrm{p}=0.044)$, (2) relación directa entre innovación en desarrollo de productos y procesos de negocio con las exportaciones reportadas en los años 2015 ( $r=0.782, p=0.038)$ y 2019 ( $r=0.764, p=0.046)$.

\section{DISCUSIÓN}

Luego de evaluar la incidencia de la innovación en la exportación de joyas de plata en siete de las principales empresas de Perú que exportaron durante los años 2015 al 2019, se encontró ausencia de relación entre ambas variables, al considerar los resultados globales tanto de exportaciones como de las puntuaciones generales de innovación.

Una de las limitaciones que presenta la investigación es el número reducido de empresas evaluadas. Sin embargo, es importante remarcar que esta muestra produjo el $67 \%$ del total de exportaciones en el periodo de estudio, lo que suma en la representatividad de los resultados.

$\mathrm{Al}$ evaluar la incidencia de innovación en producto en la exportación de joyas de plata en el Perú, se encontró ausencia de relación, tanto para los componentes bien como servicio. Lo cual quizá se deba al excesivo optimismo de las empresas por la permanencia del recurso natural (plata) de calidad en nuestro territorio. Eso sería preocupante, dado que, en el mundo hay evidencias que algunos países no sólo innovan en producto, sino que a raíz de conocimiento científico son capaces de generar minerales, tal como se ha evidenciado en Tokio por (Businesswire, 2018), quien demostró a través de una innovación en laboratorio mediante técnicas físico-químicas, el cultivo de diamantes que son visualmente idénticos a los naturales, considerando que la innovación no sólo cubre vender un elemento valioso, sino, aportar valor a la sociedad respetando el medio ambiente. De igual modo (Marinho \& Reis, 2019) señalan la viabilidad de extraer minerales del espacio, como una razonable propuesta, ejecutable y beneficiosa para la humanidad y el medio ambiente. Esto como proyección a que algún día en nuestro territorio los minerales como la plata se escasearán.

Cuando se analizó la incidencia de la innovación en procesos comerciales en la exportación de joyas de plata en Perú, se encontró relación directa entre innovación en tecnología de información, en el ítem base de datos y procesamiento de datos, con las exportaciones reportadas en el año 2016. A medida que las empresas indicaron un mayor uso de sus bases de datos y su procesamiento, particularmente en la aplicación de técnicas de minería de datos para identificar oportunidades de desarrollo empresarial, al mismo tiempo se incrementaron las exportaciones en dicho año, lo cual indica el rol fundamental que tiene el manejo de los datos en las empresas en relación a sus exportaciones.

Una opinión del Comité de Gestión de la Calidad (CGC, 2020), en relación al uso de datos y análisis de datos, además de considerarse el "cerebro" para el alineamiento de las operaciones con los objetivos estratégicos de las organizaciones, permite entender el desempeño pasado y actual detectando patrones y relaciones en datos para tomar decisiones a futuro. Así mismo, permite a la organización enfocar su inversión de talento y dinero destinado a investigación y desarrollo en soluciones personalizadas que respalden sus requisitos y los procesos que lo diferencian en el mercado nacional e internacional.

Para Collier y James (2016) estamos en la era donde las empresas se deben enfocar en los datos y el análisis. La globalización ha cambiado la manera en que las empresas hacen negocios en el comercio internacional; en tal sentido, los autores reafirman la importancia del hallazgo reportado en nuestra investigación. Dado que, refleja que la innovación en los procesos comerciales, mediante el uso de tecnología basada en la información derivada de las bases de datos y procesamiento de datos, inciden en la exportación de joyas de plata durante el año 2016.

Curiosamente, tal relación entre innovación y exportación obtenida en el año 2016 no se reflejó en los años restantes (2015, 2017-2019), lo cual podría explicarse porque las empresas 
no ejecutan de forma sistemática el uso de sus datos y el procesamiento de datos con fines de exportación. Por tal motivo, los resultados de la presente investigación adquieren mayor relevancia. Dado que reflejan la importancia de considerar de forma metódica el uso de datos y procesamiento de datos. Asimismo, abre la necesidad de realizar futuras investigaciones que profundicen en el área.

Nuestro estudio también encontró relación directa entre la innovación en desarrollo de productos y procesos de negocio con las exportaciones reportadas en los años 2015 y 2019. Tal relación podría explicarse por el comportamiento de algunas empresas que, de algún modo esos años se alinearon a la demostración cronológica de (Collier y James 2016), el mismo que remarca la importancia de considerar un área con enfoque de desarrollo de productos y otros procesos empresariales, como es la innovación en productos y procesos de negocio; aspecto planteado recientemente en el manual de Oslo. (OCDE, 2018), para medir el comportamiento de las empresas que organizan actividades de innovación por proyecto. En el manual se menciona también como articulan las innovaciones en las organizaciones. Así, por ejemplo. Una innovación en procesos puede conducir a una mejora significativa en la calidad del producto, lo que resulta en innovación tanto en el producto como en el proceso comercial; así mismo, una innovación en producto requiere del apoyo de una innovación de proceso comercial y para ser utilizable esté a la vez requiere el desarrollo de servicios como el Internet y Tecnologías de la Información y la Comunicación (TIC); y si esta función permite la creación de un nuevo canal de ventas, también puede constituir una innovación de marketing; por lo tanto, la tipología de estas innovaciones no son una clasificación en tipos mutuamente excluyentes.

Nuestros resultados se reafirman con lo argumentado por Muñoz (2016), quien indica que el trabajador (joyero) podría ser reemplazado por la maquinaria (impresora 3D, 4D u otra.) pero lo que no podrá ser reemplazado aún es el manejo de innovaciones en manufactura digital. Esta información se complementa con lo que mencionan (Collier y James, 2016), la manufactura está regresando y una de las razones serían los avances significativos en tecnología.
A pesar que, durante los años 2016 al 2018 no se encontró relación estadísticamente significativa entre la innovación por el desarrollo de productos y procesos de negocio con las exportaciones, fue evidente que durante dichos años se mostraron los coeficientes de correlación más altos reportados en el estudio, mostrando una fuerte tendencia a la presencia de relación, lo cual podría ser ratificado por estudios posteriores.

Entre los aspectos considerados en la innovación para el desarrollo de productos y procesos de negocio; destacan que la empresa cuenta con un organigrama de actividades que detalla suministro, almacenamiento, custodia, comunicación y difusión de información, lo cual denota la importancia de realizar un trabajo organizado y metódico en la empresa; asimismo, indican que la empresa hace uso de tecnología digital, que le permite hacer análisis de reacciones de los clientes con respecto a los productos que comercializa; lo cual concuerda con lo reportado por Schwab et al. (2019), quienes evidenciaron resultados positivos en ventas de joyas, luego del análisis, mediante el uso de la tecnología digital, de los consumidores sobre el desarrollo de productos y procesos en relación a electro plateado de escapularios, demostrando la convicción de usar el accesorio de plata a través de factores ambientales vinculados a la salud humana.

Otro aspecto considerado en la innovación de desarrollo de productos y procesos de negocio se refiere a si la empresa destina personal de ingeniería para desarrollar procesos de negocios a futuro. Al respecto, en nuestros resultados se evidencia que el contar con personal capacitado para desarrollar procesos de negocio, genera resultados positivos en las exportaciones. Este hallazgo se alinea a lo encontrado por (Faitaihi, 2014), quien resalta que el contar con recurso humano capacitado en innovación, es un factor que influye en el proceso de globalización de una joyería para realizar cambios en productos o procesos del negocio.

Un último punto inmerso en la innovación por el desarrollo de productos y procesos de negocio es sobre si la empresa genera y evalúa continuamente ideas para los nuevos productos, el mejoramiento del producto y de los servicios 
para satisfacer las necesidades de los clientes. En este sentido, en nuestro estudio se verificó también que la innovación de desarrollo de productos y procesos de negocio con ayuda de la tecnología incidió en las exportaciones durante los años 2015 y 2019. En relación con eso, se debe considerar también que el mejoramiento de productos y procesos de negocios requiere considerar la mejora continua (calidad), la misma que al apoyarse en la tecnología digital permite el registro de experiencia, que sumada a la creatividad humana propicia la innovación en las empresas para atender necesidades de sus clientes y ser más competitivos en el mercado internacional.

\section{CONCLUSIONES}

Si bien desde un punto de vista general no se halló relación, algunos resultados indicaron que aumentarían las exportaciones a medida que se considere tecnología de información en relación al uso de datos y procesamiento de datos. El contar con accesos a base de datos contribuye a mejorar los procesos comerciales basados en la información y el conocimiento, indicando la necesidad de que las empresas apliquen técnicas de minería de datos con el propósito de identificar oportunidades de desarrollo empresarial con fines de ser más competitivos en exportación.

Así también, los resultados indicaron que aumentarían las exportaciones a medida que la empresa desarrolle productos y procesos de negocio, donde se considere que: a) la empresa cuente con un organigrama de actividades que detalle suministro, almacenamiento, custodia, comunicación y difusión de información, b) la tecnología digital a la que accede la empresa le permita hacer análisis de reacciones de los clientes con respecto a los productos que comercializa, c) la empresa genere y evalúe continuamente ideas para los nuevos productos, el mejoramiento del producto y de los servicios para satisfacer las necesidades de los clientes, d) la empresa destine personal de ingeniería para desarrollar procesos de negocios a futuro.

Finalmente, podemos concluir que, la innovación constante en los ítems mencionados anteriormente es esencial para tener mayor probabilidad de éxito en mercados globales.

\section{RECOMENDACIONES}

Es necesario mencionar que las empresas de los países que han creado herramientas para la innovación, manejan conceptos que facilitan la innovación. En ese contexto, las empresas exportadoras de joyería de plata de Perú se deberían alinear, a través de investigaciones que generen conocimientos sobre. El manejo de datos, utilizando tecnología digital en sus procesos; de modo que administren sus empresas con enfoque en la era de los datos y el procesamiento de datos. Así mismo, consideren en su estructura organizacional un área con enfoque en desarrollo de productos y otros procesos de negocio a futuro.

Se recomienda también a las empresas que estén agrupadas como sector en un gremio que tenga representación internacional, donde además de adecuarse a los lineamientos y cadenas de valor de sus socios comerciales, consideren que, para competir en el mercado internacional es necesario contar con una cadena de valor que concentre empresas, Centros de Innovación Tecnológica (CITES), institutos, universidades, con una rápida adecuación u homogenización curricular en las carreras afines que faciliten el uso de innovación tecnológica con enfoque en bases de datos y procesamiento de datos. Del mismo modo se recomienda realizar una siguiente investigación que tenga como base la investigación concluida, que ayude a profundizar y fortalecer desde un punto de vista científico en dicho sector.

Es menester recordar que esta adecuación no se resuelve aisladamente, es importante la actuación del sector público en el rol de facilitador al desarrollar capacidades y aprendizaje al interior de las empresas, mejorar la articulación interinstitucional. De modo especifico, es necesario políticas que faciliten el intercambio comercial tecnológico, fijando metas y objetivos apoyados en las alianzas comerciales, con países de nuestra región y a la vez alineados a la visión de países innovadores líderes en el comercio internacional. Por otro lado, es necesario realizar estudios de investigación que evalúen dichas políticas que permitan el desarrollo de una cultura de mejora continua propicia para la innovación en procesos comerciales que apoyen en las exportaciones de joyas de plata en el Perú. 


\section{REFERENCIAS BIBLIOGRÁFICAS}

Banco Mundial. (06 de enero de 2020). Crecimiento del PBI. Percápita. http://wdi.worldbank.org/ table/4.2.

Businesswire. (10 de septiembre de 2018). Tecnología Pure Diamond Block Chain - Innovación revolucionaria de la industria de la joyería. https://www.businesswire.com/news/ home/20180822005212/es/

Cámara de Comercio de Bogotá. (8 de noviembre de 2020). Cluster de Joyería y Bisutería.https://www.ccb.org.co/Clusters/Cluster-de-Joyeria-y-Bisuteria.

Cámara de Comercio de España. (8 de diciembre de 2020). Innovación. https://www.camara.es/ innovacion-y-competitividad/como-ser-competitivo\#itinerario52

Cámara de Comercio de Lima. (5 de noviembre de 2020). XV Cumbre Internacional de Comercio exterior. https://www.facebook.com/CamaradeLima/videos/377039996985515

Collier, D., \& James, E. (2016). Administración de operaciones. Cengage Learning.

Comité de Gestión de la Calidad. (2020). Bases premio nacional a la calidad 2020.

Rachel N. (20 de marzo de 2014). mundo - noticias. Corporación Británica de Radiodifusión. Recuperado el 10 de enero del 2020 de: https://www. bbc.com/mundo/noticias/2014/03/140325_ciencia_recursos_minerales_escasos_np

https://webcache.googleusercontent.com/search?q=cache:nVc3hh3yv6EJ:https://www.bbc. com/mundo/noticias/2014/03/140325_ciencia_recursos_minerales_escasos_np $+\& c-$ $\mathrm{d}=1 \& \mathrm{hl}=\mathrm{es}-419 \& \mathrm{ct}=\mathrm{clnk} \& \mathrm{gl}=\mathrm{pe}$

Faitaihi, M. A. (2014). Factores que influyen en el proceso de globalización de una marca de joyería. (Tesis de doctorado, Universidad Walden). Repositorio Institucional UNMSM. https:// search.proquest.com/docview/1640934517?accountid $=12268$

Frohmann, A., Mulder, N., Olmos, X., \& Urmeneta, R. (2016). Internacionalización de las pymes: innovación para exportar. Comisión Económica para América Latina y el Caribe https://repositorio.cepal.org/bitstream/handle/11362/40737/1/S1600442_es.pdf

Malhotra, N. (2008). Investigación de mercados. Pearson educación.

Marinho Amorim, H., \& Reis Rochael, C (2019). Minerales espaciales: cosas de nadie en beneficio de todos. Derecho PUCP, (83), 89 -131. https:// doi.org/10.18800/derechopucp.201902.004

Ministerio de Comercio Exterior y Turismo. (2013). Plan Estratégico Nacional Exportador 2025. https://www.mincetur.gob.pe/wp-content/ uploads/documentos/comercio_exterior/ plan_exportador/Penx_2025/PENX_FINAL_101215.pdf

Muñoz Mesa, L. y Sánchez Trujillo, J. H. (2016). El impacto de la impresión 3D en la joyería. Lámpsakos, (16), 89-97. DOI: http://dx.doi. org/10.21501/21454086.1969

Organización para la Cooperación y el Desarrollo Económico. (2018). Directrices para recopilar, informar y utilizar datos de innovación, Manual de Oslo 2018 (4. - ed.): http://www.oecd.org/science/oslo-manual-2018-9789264304604-en.htm.

Oppenheimer, A. (2006). Cuentos Chinos: El tema no es libre comercio, sino la competividad. Verlap S.A.

Organización para la Cooperación y el Desarrollo Económico. (2019). Perspectivas económicas de América Latina 2019-desarrollo en transición. https://www.oecd.org/dev/americas/Overview_SP-Leo-2019.pdf.

Porter, M. (2014). Ser competitivo: Las 5 fuerzas competitivas que moldean la estrategia de las empresas. Deusto.

Ratten, V. (2015). El papel cambiante de la organización del aprendizaje y la gestión del conocimiento en el entorno internacional. Thunderbird international business review. https://doi. org/10.1002/tie.21657

Schwab, F., Jacques, J. y Branca F, O. (agosto de 2019). Joyería galvanizada de plata 925 y oro de $18 \mathrm{k}$ : Un análisi comparativo de impactos ambientales. International Journal of Advances in Engineering \& Technology; Bareilly Tomo 12. https:// search.proquest.com/docview/2309751513/ fulltextPDF/D207F39E7B4F488CPQ/1?accountid $=12268$.

Serrano Camarena, D. E., Amparo Tello, D. y Contreras Teodoro, A. B. (2020). Reconfiguración de las redes productivas en la industria de la joyería en Thrissur, India, Chantaburi, Tailandia y Guadalajara, México. Revista mexicana de estudios sobre la Cuenca del Pacífico. 14 (28). http:// revistasacademicas.ucol.mx/index.php/portes/ article/download/2069/2251.

Servicio Geológico de los Estados Unidos. (2020). Hoja de datos de plata. https://pubs.usgs.gov/ periodicals/mcs2020/mcs2020-silver.pdf 
Superintendencia Nacional Tributaria. (2019). In- Trade Map. (20 de abril de 2020). Estadísticas del formación Arancelaria. http://www.aduanet. comercio para el desarrollo internacional de gob.pe/itarancel/arancelS01Alias

Superintendencia Nacional Tributaria. (2020). Orientación aduanera. https://www.sunat.gob.pe/ orientacionaduanera/exportacion/index.html las empresas. Datos comerciales mensuales, trimestrales y anuales. Valores de importación y exportación, volumenes, tasas de crecimiento, cuotas de mercado, etc. https://www.trademap.org/Index.aspx 